\title{
Quantum Mechanics Analysis: Modeling and Simulation of some simple systems
}

\author{
Younis A. Shah ${ }^{\mathrm{a}}$, Irshad.A. Mir ${ }^{\mathrm{b}}$, Uzair M. Rathe $\mathrm{a}^{\mathrm{a}, \mathrm{b}, *}$ \\ ${ }^{a}$ Department of Computer Science, University of Kashmir, Srinagar, 190006, India \\ ${ }^{b}$ Department of Computer Science, University of Kashmir, Srinagar, 190006, India \\ ${ }_{a, b *}$ Software Engineer, BQE Inc., Srinagar, 190001 India
}

\begin{abstract}
Quantum Mechanics is a radically new way of thinking about the Nature. It is used for explaining the behavior of an electron to the possible existence of parallel universes. Most of the times, it is just too difficult to get an intuitive grasp of Quantum Mechanics. Quantum Mechanics is a mathematical framework for describing atomic and subatomic systems. In this paper the basic principles of Quantum Mechanics have been presented. It has been shown that classical probabilities when added increase the overall probability while in quantum probabilities can cancel each other and hence lower the overall probability. A simple approach has been taken by modeling the systems as graphs. The states and dynamics of the systems have been modeled as matrices. The principle of Superposition and the phenomenon of Interference have been explained in a single simulation. Finally the BLAS functions of Julia programming language have been used for simulating the systems under study on a classical computer and a detailed discussion is presented to highlight the incapability of the classical explanation of quantum phenomenon.
\end{abstract}

Index Terms: Classical Deterministic Systems, Classical Probabilistic Systems, Quantum Systems, Julia, Quantum Computing, Superposition Principle.

(C) 2016 Published by MECS Publisher. Selection and/or peer review under responsibility of the Research Association of Modern Education and Computer Science

\section{Introduction}

Classical systems are those which obey the laws of classical physics [1] (Susskind and Hrabovsky G, 2014). They can be categorized as classical deterministic systems and classical probabilistic systems. The former has a state space as a mathematical set and laws governing the time evolution of the system are deterministic. The latter evolves with time in a probabilistic way and if the classical probabilities are added

* Corresponding author.

E-mail address: younisashah@live.in,iamir@uok.edu.in 
they increase the total probability [1] (Susskind and Hrabovsky G, 2014). However, Quantum systems are different from both of these systems. In addition to being in one of the states of the state space, a quantum system can be in a superposition of all the states of its state space. Also, quantum probabilities are different from classical probabilities. While classical probabilities are increase the probability when added, quantum probabilities when added can lower the overall probability. This is referred to as Quantum Interference[2] (Yanofsky et al, 2008). We'll describe all these concepts in this paper with the following scheme. Section 2 presents the preliminaries and background on each of the three systems. Section 3 introduces the three systems we'll be simulating. Section 4 describes the actual simulations performed. Section54 presents the results and plots of the simulations. Section 6 discusses the results and plots obtained. Finally section 7 concludes the paper.

\section{Preliminaries and Background}

This section explains the classical deterministic and probabilistic system and Quantum systems in a very lucid manner. The states space and how the system changes with time have been explained.

\subsection{Classical Deterministic Systems}

Classical systems obey the laws of classical physics [1] (Susskind and Hrabovsky G, 2014). Classical particles like marbles, bullets, grains of sand etc., constitute classical systems. Classical particles assemble together to form larger classical systems like a simple dice, a lever-pulley system, Solar System and even the traditional personal computer (henceforth referred to as classical computer). Every classical system has a state - the condition or configuration of the system e.g. trivial classical system is a coin. A coin has two states Head $(\mathrm{H})$ or Tail $(\mathrm{T})$. Another two-state classical system is a binary digit (bit) - 0 or 1[3] (Mermin, N. David, 2003). The mathematical set of all the states of a classical system is called its State Space[1] (Susskind and Hrabovsky G, 2014). A classical system evolves with time. A classical system whose state changes with time is called a classical dynamical system. A dynamical system not only has a State Space but also is characterized by a law that describes how the system evolves with time. Such law is referred to as a law of motion or dynamical law. This law simply tells the next state given the present state. A simple dynamical law for a coin system can be stated as

$$
\text { Next State }=\left\{\begin{array}{l}
H, \text { if present state is } T \\
T, \text { if present state is } H
\end{array}\right.
$$

To write the dynamical law as an equation, the variables describing the systems need to be identified. These variables are called its degrees of freedom. A two-state bit system or a coin system has one degree of freedom, denoted by $\boldsymbol{D}$. The possible values of $\boldsymbol{D}$ are 1 (H or binary 1 ) or 0 (T or binary 0 ). The dynamical law can be written as [1] (Susskind and Hrabovsky G, 2014):

$$
D(n+1)=1-D(n) ; \text { at } n^{\text {th }} \text { time instant. }
$$

Together with the state information of the system and its dynamical law, all the future of the system can be predicted with certainty. Such a classical system is referred to as a classical deterministic system. Sometimes it is just too difficult to formulate a dynamical law of a classical system as an equation. These systems can usually be modeled as a directed graphs, with directed edges giving the "way" the system evolves with time the dynamical law. The dynamical law can be expressed as an adjacency matrix (Grimaldi, 2003) - the dynamics matrix [2] (Yanofsky et al, 2008), [4] (Arrighi, Pablo, 2003). The state of such a system can also be described by a matrix - a column vector [2] (Yanofsky et al, 2008). 


\subsection{Classical Probabilistic Systems}

The world, as perceived by us, is deterministic: it has deterministic states and is governed by deterministic laws which can tell us what its past was and future will be. Hence the role of probability in describing any physical (classical) phenomena is subtle [5] (Bao Lei, and Edward F. Redish, 2002). A system consisting of a large number of particles or a system which has a large number of degrees of freedom is very difficult to analyze and it is not practical to obtain or store the knowledge (state and dynamics) of every particle of the system. In such circumstances, probability theory is used to describe the state and dynamics of the overall system [6] (Boas, Mary L, 2006). Consider a classical system consisting of a mass attached to a spring which in turn is attached to a wall as shown in Fig 1. The mass $\mathrm{m}$ (with a midpoint shown as a white dot), has a well-defined path, moving along the horizontal $\mathrm{x}$-direction.

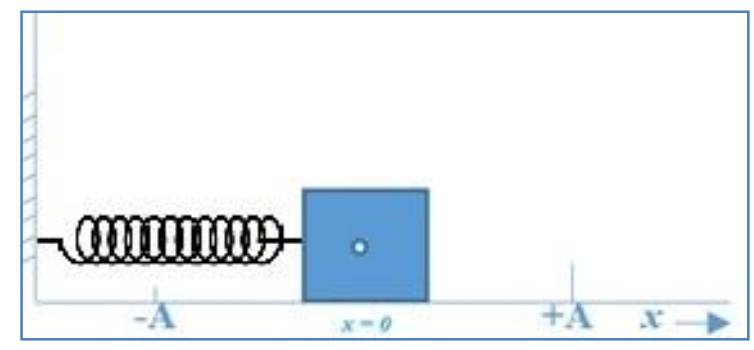

Fig.1. A spring-mass system moving in $\mathrm{x}$-direction between points $(-\mathrm{A})$ and $(+\mathrm{A})$.

Irrespective of the fact that classical particles have well-defined path, we can still formulate a classical probabilistic density function which roughly gives us the dynamical law of the system. Consider the above spring-mass system. Let us assume when we observed the system, it was already in the state of motion. The goal is to find the dynamics of the system. We begin by taking a number of photographs of the mass or particle (its midpoint actually) at random times. The probability of the finding the particle at a given position is proportional to the time spent by the particle at that position. This means more the number of photographs of the particle at a particular position, more time it spends there. At the points, $(-\mathrm{A})$ and $(+\mathrm{A})$, the particle is momentarily at rest, hence the probability of finding the particle at these two points is more (maximum, actually) as compared to the position $\mathrm{x}=0$, where it is minimum. From these probability values, we can formulate a probabilistic dynamical law of the system [7] (Simulation 18, 2014). A trivial classical probabilistic system is a classical coin. The result of flipping the coin is either Heads (T) or Tails $(\mathrm{H})$. We just don't know what the state of the coin will be after flipping - probably $\mathrm{H}$ or probably $\mathrm{T}$ (exclusive OR). This demonstrates our partial ignorance of the state of system. The probability theory tries to quantify this ignorance of the state by saying in one flip of coin, there is $1 / 2$ chance or getting an $\mathrm{H}$ and $1 / 2$ chance of getting a T[6] (Boas, Mary L, 2006).

\subsection{Quantum Systems}

As seen, classical systems can be described using deterministic laws as well as using probabilistic laws. Quantum systems are, however, different in the sense they are inherently non-deterministic [2] (Yanofsky et al, 2008). A new mathematical framework needed to be developed in order to understand quantum systems.

Explanation of quantum systems using the physical laws of classical mechanics led to absurd results and contradictions [7] (Simulation 18, 2014). In order to understand the basics of Quantum systems, we will consider the following example of a subatomic particle; say an electron, occupying one of the $\left\{x_{0}, x_{1}, \ldots, x_{i}, \ldots, x_{n-1}\right\}$ levels, as shown in Fig 2. 


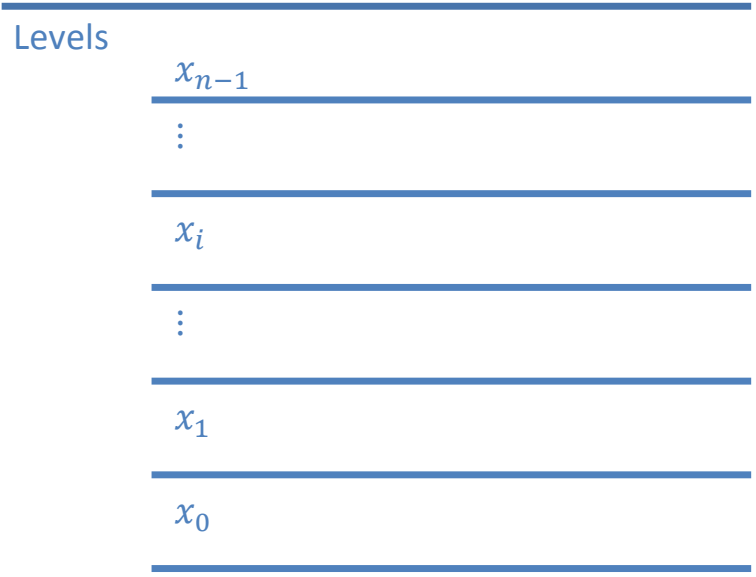

Fig.2. The $n$ levels which can be occupied by the subatomic particle.

The particle can be found in one of the states (levels) $x_{i}$. This can be symbolized by $\left|x_{i}\right\rangle$. This notation was introduced by Dirac and is called a ket vector [2] (Yanofsky et al, 2008). Just like the state of a classical system can be described by an n-dimensional column vector, the state of a quantum system can be described by an n-dimensional complex column vector $\left[c_{0}, c_{1}, \ldots, c_{n-1}\right]^{T}$. For each of the basic states $\left|x_{i}\right\rangle$, a column vector can be associated with it, given as $\left|x_{0}\right\rangle \rightarrow[1,0, \ldots, 0]^{T},\left|x_{1}\right\rangle \rightarrow[0,1, \ldots, 0]^{T} \ldots\left|x_{i}\right\rangle \rightarrow[0,0, \ldots 1, \ldots, 0]^{T} \ldots\left|x_{n-1}\right\rangle \rightarrow[0,0, \ldots, 1]^{T} . \quad$ Unlike classical particles, a quantum mechanical particle can be in multiples levels at the same time. This may seem counter-intuitive. An arbitrary quantum state is a linear combination of the basis states $\left|x_{i}\right\rangle \forall i \leq n-1$. This is written as follows:

$$
|\psi\rangle=c_{0}\left|x_{0}\right\rangle+c_{1}\left|x_{1}\right\rangle+\cdots+c_{n-1}\left|x_{n-1}\right\rangle=\sum_{i=0}^{n-1} c_{i}\left|x_{i}\right\rangle
$$

The complex numbers $c_{i} \forall i \leq n-1, c_{i} \in C$ are called probability amplitudes. Equation (3) tells that, every quantum state can be represented by an n-dimensional complex vector $\left[c_{0}, c_{1}, \ldots, c_{n-1}\right]^{T}$. The state $|\psi\rangle$ is the superposition of all the basis states $\left|x_{i}\right\rangle$. Physically, the superposition of states means that the particle is simultaneously in all the states at the same time [2] (Yanofsky et al, 2008), [9 (Yanofsky, Noson S, 2011), ][3] (Mermin, N. David, 2003). Quantum mechanics tells us that an "unobserved" particle in a superposition state, when observed, will "collapse" to one of the basic states. Hence, a particle in superposition state $|\psi\rangle$ when observed will be found in one of the basic state $\left|x_{i}\right\rangle$ CITATION PAM08 V 1033 [10] (Dirac, Paul, 1981). Just like we can vary the quantity of basic ingredients (like eggs, cocoa powder, espresso powder, etc.) to make a chocolate cake [11] (Robyn The Best Chocolate Cake Recipe, 2015) to obtain different flavors, similarly, there are different superposed or "blended" states. The coefficients, $c_{i}$ of the state $|\psi\rangle$ tell us precisely in what superposed state the particle is in. The probability that the particle will be found in one of the levels is given by:

$$
P\left(x_{i}\right)=\frac{\left|c_{i}\right|^{2}}{|| \psi\rangle\left.\right|^{2}}=\frac{\left|c_{i}\right|^{2}}{\sum_{i}\left|c_{i}\right|^{2}}
$$

Quantum systems are different from classical systems. This can be illustrated by a simple game where we have a ball and two cups. Classically, the ball can be in one of the cups but quantum mechanically, the ball will be in both the cups simultaneously [12] (Biamonte Jacob D, 2014). 


\subsection{A Description of General Physical Systems}

A physical system can be in one of the many states which are intrinsic to the system ( $\mathrm{H}$ or $\mathrm{T}$ are intrinsic to a coin system. It can't be in any other states). Hence, a system is associated with a set of states. This can be easily represented as a column vector - a state vector. For example, if a particle is moving on a plane surface with some speed such that for every 10 meters it covers, we note its speed. So the spliced "speed" vector, $S$ for a distance of 50 meters can be represented as $S=[10,12,6,11,23]^{T}$. Similarly, a common salt crystal ( $\left.\mathrm{NaCl}\right)$ [13] (Earley Clarke, 2008) can be modeled as a lattice such that the lattice cells contain a Sodium (Na) or a Chlorine $(\mathrm{Cl})$ particle. Either a cell contains (1) a particle (say $\mathrm{Cl}$ ) or not (0). This can be represented (for the top layer of the standard $\mathrm{NaCl}$ lattice - boundary atoms only) in the following spliced form $C=[\mathbf{1}, \mathbf{0}, \mathbf{1}, \mathbf{0}, \mathbf{1}, \mathbf{0}, \mathbf{1}, \mathbf{0}]^{T}$. The state of physical system also evolves with time using certain rule(s). These rules are the laws of physics for the given set of states of a physical object. These rules, when applied to the state vector, produce a new state vector which represents the new state of the physical system. In other words, these laws transform the states into new states over (discrete) time steps [14] (Leornard Susskind, 2014). These laws or rules can be written in the form of matrices. Consider a 2-bit system. The state space of a 2-bit system is $\{\mathbf{0 0}, \mathbf{0 1}, \mathbf{1 0}, \mathbf{1 1}\}$. Some of the rules can be (Fig.3)

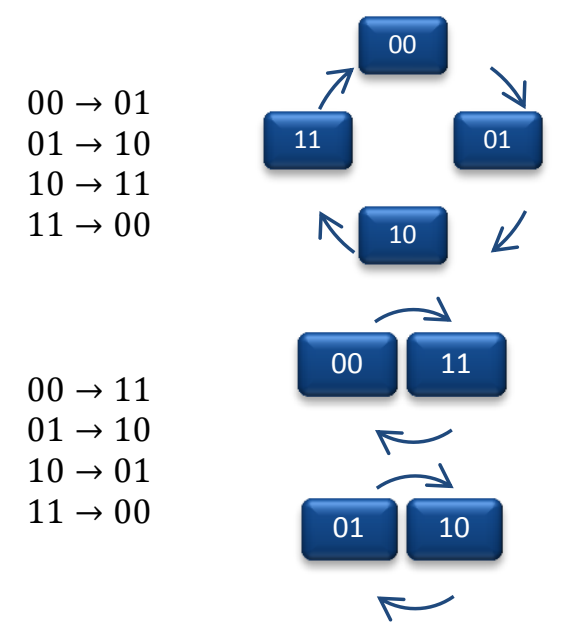

Fig.3. State Space of a 2-bit System.

Consider another example of a (classical) physical system consisting of a particle and four positions it can occupy. The particle can occupy one of 4 possible positions. The system is shown in Fig 4.

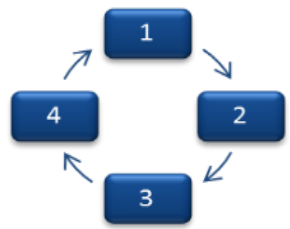

Fig.4. A classical system of a particle and one of the 4 positions it can occupy

Let a column vector $P$ represents the state of the system - where (position) the particle is. 


$$
P=\left[\begin{array}{l}
p_{1} \\
p_{2} \\
p_{3} \\
p_{4}
\end{array}\right] \text { where } p_{i} \in\{0,1\} \forall i \in\{1,2,3,4\}
$$

$\boldsymbol{P}$ is such that if the particle is at position $\boldsymbol{i}$, then $\boldsymbol{p}_{\boldsymbol{i}}$ is 1 , and rest of $\boldsymbol{p}_{\boldsymbol{j}}{ }^{\prime} \boldsymbol{s}$ are 0 . Then, the set of possible states of the system are:

$$
\left[\begin{array}{l}
1 \\
0 \\
0 \\
0
\end{array}\right],\left[\begin{array}{l}
0 \\
1 \\
0 \\
0
\end{array}\right],\left[\begin{array}{l}
0 \\
0 \\
1 \\
0
\end{array}\right],\left[\begin{array}{l}
0 \\
0 \\
0 \\
1
\end{array}\right]
$$

The next step is to figure out the law of physics for the system or the rule for transforming the present state to a next state. The law can be represented by a $4 \times 4$ matrix $\boldsymbol{M}$.

$$
M=\left[\begin{array}{llll}
0 & 0 & 0 & 1 \\
1 & 0 & 0 & 0 \\
0 & 1 & 0 & 0 \\
0 & 0 & 1 & 0
\end{array}\right]
$$

We will call $M$, the dynamics matrix of the system. The columns of $M$ correspond to the each position of the system. The first column transforms the present state of the system to state given by position 2, the second column transforms the state of the system to the state given by position 3, and so on [14] (Leornard Susskind, 2014). Applying the dynamics matrix to a state vector gives the next state of the system. This can be easily seen by multiplying $M$ with the state vector. If the system is in state, say $[1,0,0,0]^{T}$, then, the next state should be $[0,1,0,0]^{T}$. This can be verified as follow:

$$
M *\left[\begin{array}{l}
1 \\
0 \\
0 \\
0
\end{array}\right]=\left[\begin{array}{llll}
0 & 0 & 0 & 1 \\
1 & 0 & 0 & 0 \\
0 & 1 & 0 & 0 \\
0 & 0 & 1 & 0
\end{array}\right] *\left[\begin{array}{l}
1 \\
0 \\
0 \\
0
\end{array}\right]=\left[\begin{array}{l}
0 \\
1 \\
0 \\
0
\end{array}\right]
$$

Multiplication of $\mathrm{M}$ with the state vector $\mathrm{n}$ times, gives us the state of the system after $\mathrm{n}$ discrete time units [2] (Yanofsky et al, 2008). Thus, the time evolution of a system is simply the successive application ofthe dynamics matrix to the state vector [14] (Leornard Susskind, 2014).

\section{Simulation Setup}

In this section we will present the systems on which we will be performing our simulations and generating our results. We will use graphs to model these systems [15] (Grimaldi, Ralph P, 2006). These systems are implemented using Julia Programming Language.

\subsection{Classical Deterministic Systems}

In classical deterministic system we will be using 10 classical particles scattered on a 5-vertex graph. The state of such a system - the state vector, will be represented by the column vector. Each element of the column vector will represent the number of classical particles at some vertex at some point in time. The laws of physics 
of system (time evolution of system) - the dynamics matrix will be represented using an adjacency matrix [15] (Grimaldi, Ralph P, 2006). If, for example, we may have to determine the state after 5 time units, it can be calculated by the following equation.

$$
Y=M^{5} * X
$$

Where $\mathrm{Y}$ represents the state after 10 time units, $\mathrm{M}$ represents the dynamics matrix and $\mathrm{X}$ represents the state vector. It is assumed that it takes one time unit for each classical particles to shift to another vertex if path exists.

\subsection{Classical Probabilistic Systems}

In classical probabilistic system, the system under consideration is the classic double slit experiment [9] (Yanofsky, Noson S, 2011). Taking our system into consideration, we will be having one source emitting a classical particle and 2 slits through which this particle can hit the wall. The particle that is able to pass through any of these slits will hit the wall at one of the 5 targets just behind the slits. Now the overall system will be, 1 source, 2 slits and 5 targets which allows us to conclude that the particle can be at any position at some point in time among these 8 positions. Since we have only one particle and it can occupy only one position among 8 position available, we calculate the probability of finding the particle at these position. The state of such a system - state vector, is represented by column vector. Each element of such state vector is the probability of particle to be at some position. For instance, if we have to measure the state of such system after 8 time units, we will do that by:

$$
Y=M^{8} * X
$$

Where $\mathrm{Y}$ represents the final state vector, $\mathrm{M}$ represents the dynamics matrix and $\mathrm{X}$ represents the state vector.

It is assumed that the particle takes 1 time unit to move from source to a slit and 1 time unit from the slit to a target. Also, given the number of slits, $n$, the number of targets is given by $2 n+1$. Hence, for 2 slits, we have a total of 5 targets, with 3 targets directly behind each slit and one of the targets is mutually shared by the slits.

\subsection{Quantum Systems}

In our experiment (simulation) with Quantum Systems, we will be having a photon source emitting a quantum mechanical particle, a photon and 2 slits through which this particle will pass and strike the 5 photon detectors placed right behind the slits. Our Quantum system will be 1 source, 2 slits, a photon and 5 photon detectors. The state of the system - the state vector, will be represented by a column vector in which each element will be the probability amplitude of the photon at that position. After 8 time units, the state of this Quantum System is given by

$$
Y=M^{8} * X
$$

Where $\mathrm{Y}$ represents the system state, $\mathrm{M}$ represents the dynamics matrix and $\mathrm{X}$ represents the column vector. It is assumed that the photon takes 1 time unit to move from source to a slit and 1 time unit from the slit to a photon detector. Also, given the number of slits, $n$, the number of photon detectors is given by $2 \mathrm{n}+1$. Hence, for 2 slits, we have a total of 5 photon detectors, with 3 photon detectors directly behind each slit and one of the photon detectors is mutually shared by the 2 slits.

\section{Simulation and Experiment}


We will describe the three experiments (simulations) carried out using a classical programming language, Julia on a classical computer. The simulations describe the dynamics matrix or laws of physics, the state vector and evolution of state vector with time.

\subsection{Classical Deterministic Systems}

As we discussed in section III-A, our classical deterministic system will be a "10-classical-particle, 5-vertex graph". Fig 5 depicts the system. The dynamics matrix, M, generated by the simulation is

$$
M=\left[\begin{array}{lllll}
0 & 0 & 0 & 0 & 0 \\
0 & 0 & 0 & 0 & 1 \\
0 & 1 & 0 & 1 & 0 \\
0 & 0 & 0 & 0 & 1 \\
0 & 0 & 1 & 1 & 0
\end{array}\right]
$$

The particles distribution on the vertices is as: 2 on vertex 0,4 particles on vertex 1,2 particles on vertex 2,1 particle on vertex 3 and 1 particle on vertex 4 . This initial state vector is given, $X$

The system will evolve after every time unit which is given by matrix multiplication of $\mathrm{M}$ and $\mathrm{X}$. We will input the total number of time units, $\mathrm{n}(=5)$ for which the system (state vector) evolves. After 5 time units the final state vector of the system is calculated, which is represented in following equation

$$
Y=M^{5} * X
$$

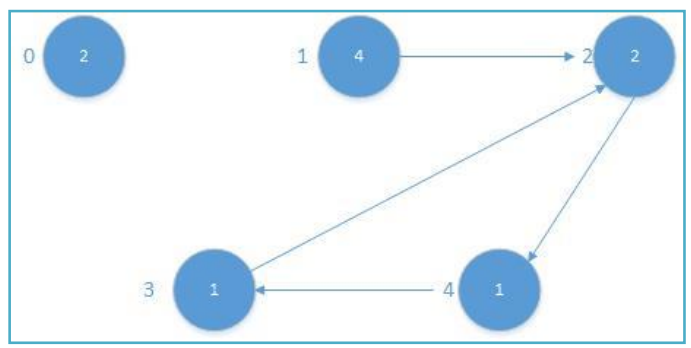

Fig.5. A 10-classical-particle, 5-vertex graph of a classical deterministic system

\subsection{Classical Probabilistic System}

As explained in section 3.2 the system under consideration is a "1-classical-particle, 8-vertex graph". Fig 6 shows the system modelled as a graph. The experiment (Julia simulation) starts by forming the dynamics matrix of the system. Hence, we'll get an 8x8 matrix, M. The dynamics matrix, M, generated by the simulation is 


$$
M=\left[\begin{array}{cccccccc}
0 & 0 & 0 & 0 & 0 & 0 & 0 & 0 \\
1 / 2 & 0 & 0 & 0 & 0 & 0 & 0 & 0 \\
1 / 2 & 0 & 0 & 0 & 0 & 0 & 0 & 0 \\
0 & 1 / 3 & 0 & 1 & 0 & 0 & 0 & 0 \\
0 & 1 / 3 & 0 & 0 & 1 & 0 & 0 & 0 \\
0 & 1 / 3 & 1 / 3 & 0 & 0 & 1 & 0 & 0 \\
0 & 0 & 1 / 3 & 0 & 0 & 0 & 1 & 0 \\
0 & 0 & 1 / 3 & 0 & 0 & 0 & 0 & 1
\end{array}\right]
$$

Initially, the particle will be on vertex 1 and with time it will be found on any of the other 7 vertices. This initial state vector is given by a column vector, $\mathrm{X}$

$$
X=[1,0,0,0,0,0,0,0]^{T}
$$

The time evolution of the system will be given by matrix multiplication of $\mathrm{M}$ and $\mathrm{X}$. Next, the simulation receives the total number of time units, $\mathrm{n}(=8)$ for which the system (state vector) evolves. The simulation then calculates the final state vector, $\mathrm{Y}$ of the system after evolving it for $\mathrm{n}(=8)$ time units.

$$
Y=M^{8} * X
$$

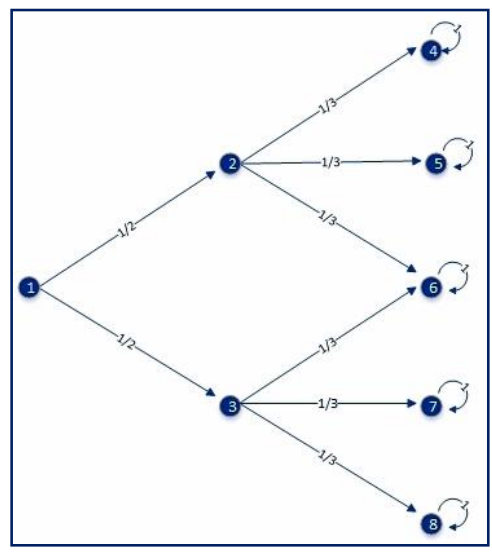

Fig.6. A 1-classical-particle, 8-vertex graph of a classical probabilistic system.

\subsection{Quantum Systems}

Here the system under consideration is a "1-photon, 8-vertex graph". Fig 7 shows the system. After forming the dynamics matrix of the system - an $8 \times 8$ matrix, $\mathrm{P}$, the simulation constructs the initial state vector, $\mathrm{X}$. At the start of the simulation, the photon will be at the source (vertex 1). Hence the initial state vector will be:

$$
X=[1,0,0,0,0,0,0,0]^{T}
$$


The dynamics matrix, $\mathrm{P}$ is

$$
P=\left[\begin{array}{cccccccc}
0 & 0 & 0 & 0 & 0 & 0 & 0 & 0 \\
\frac{1+i}{2} & 0 & 0 & 0 & 0 & 0 & 0 & 0 \\
\frac{1+i}{2} & 0 & 0 & 0 & 0 & 0 & 0 & 0 \\
0 & \frac{i}{\sqrt{3}} & 0 & 1 & 0 & 0 & 0 & 0 \\
0 & \frac{i}{\sqrt{3}} & 0 & 0 & 1 & 0 & 0 & 0 \\
0 & \frac{1-i}{\sqrt{6}} & \frac{-1+i}{\sqrt{6}} & 0 & 0 & 1 & 0 & 0 \\
0 & 0 & \frac{i}{\sqrt{3}} & 0 & 0 & 0 & 1 & 0 \\
0 & 0 & \frac{i}{\sqrt{3}} & 0 & 0 & 0 & 0 & 1
\end{array}\right]
$$

The number of time units $\mathrm{n}$ for which the simulation is run equals 8 . An important point to note here is that the above matrix is not a unitary matrix. We have kept the graph simple and ignored many paths the photon can travel. The above matrix is good enough to demonstrate the phenomena of Quantum Interference and Superposition. Again, as discussed, the time evolution of the system is given by the matrix multiplication of $\mathrm{R}$ and $\mathrm{X}$. After 8 time units, the final state vector, $\mathrm{Q}$ is given by

$$
Q=R^{8} * X
$$

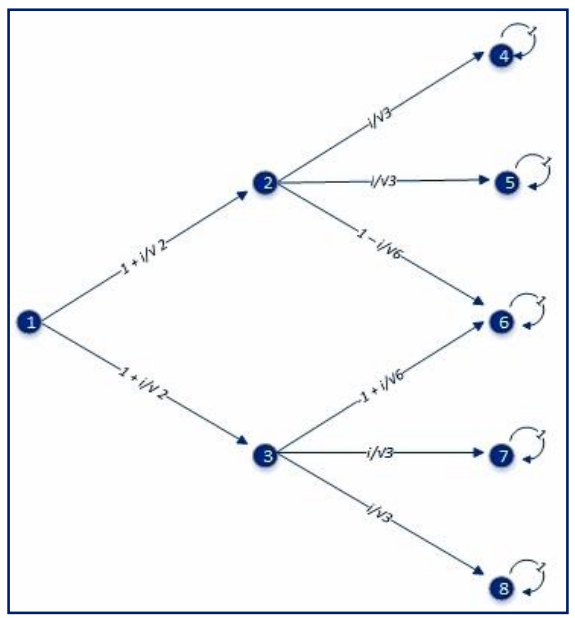

Fig.7. A 1-photon, 8-vertex graph of a quantum system.

\section{Results}

The results of simulating the previously defined systems are discussed. Here, we give the plots that were obtained by simulating these systems in Julia programming language. 


\subsection{Classical Deterministic Systems}

We ran the system for 5 time units and plotted the state vector after each time unit. Fig 8 through 12 show the time evolution of the state vector for 5 time units and Fig 13 shows the collage of the all the plots of state vectors.

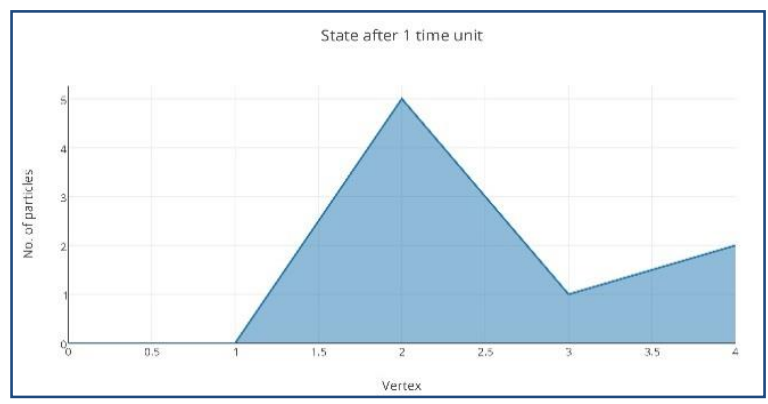

Fig.8. Plot of state vector after 1 time unit.

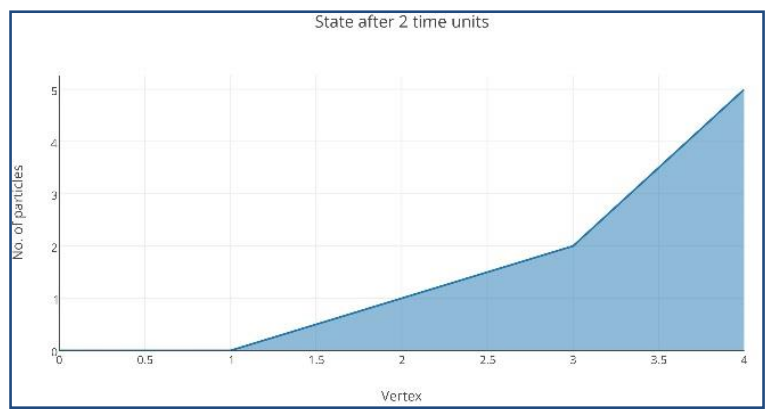

Fig.9. Plot of state vector after 2 time unit.

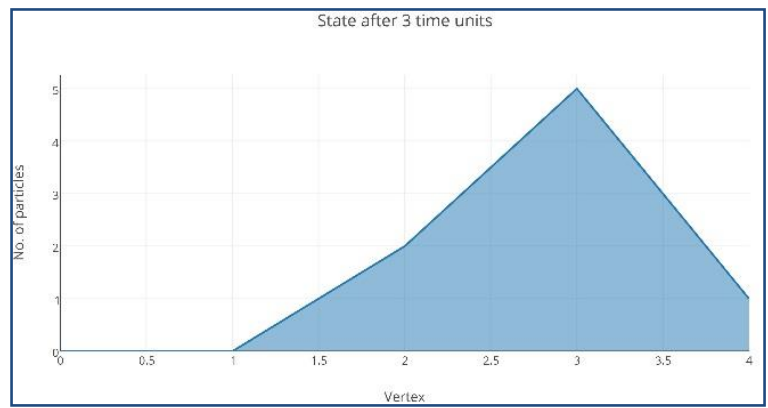

Fig.10. Plot of state vector after 3 time unit. 


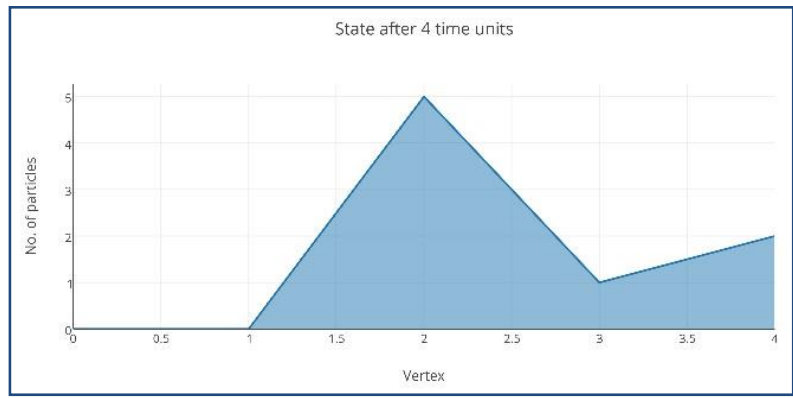

Fig.11. Plot of state vector after 4 time unit.

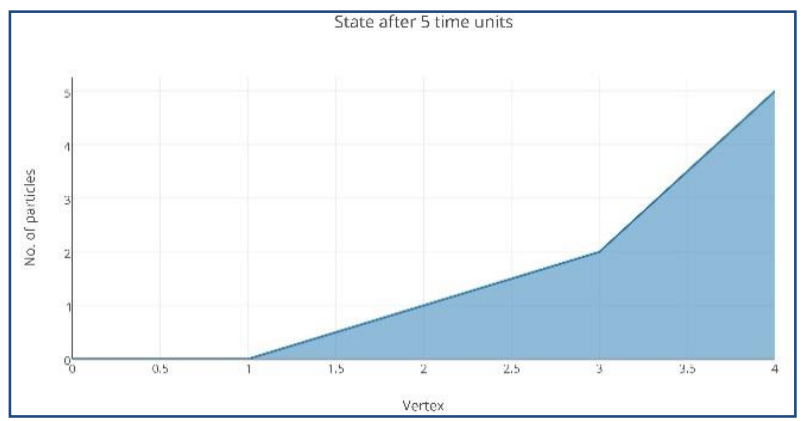

Fig.12. Plot of state vector after 5 time unit.

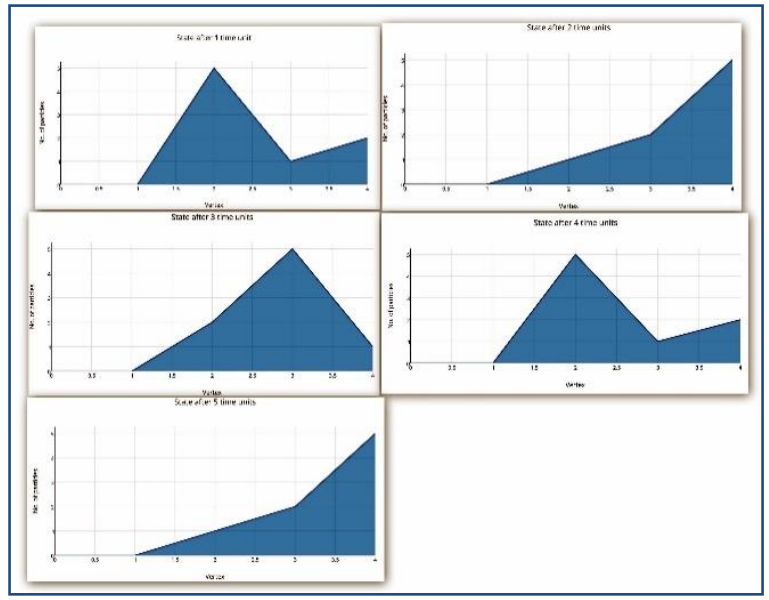

Fig.13. A collage of the plots of state vectors after each time unit

\subsection{Classical Probabilistic Systems}

We recorded the state vector of the system through its time evolution, by taking snapshots of the column vectors and plotting them. We ran the system for 8 time units and plotted the state vector after each time unit. Figures 14 and 15 show the state vector after 1 and 2 time units, respectively and Figure 15 shows the collage of the all the plots of state vectors after each time unit. 


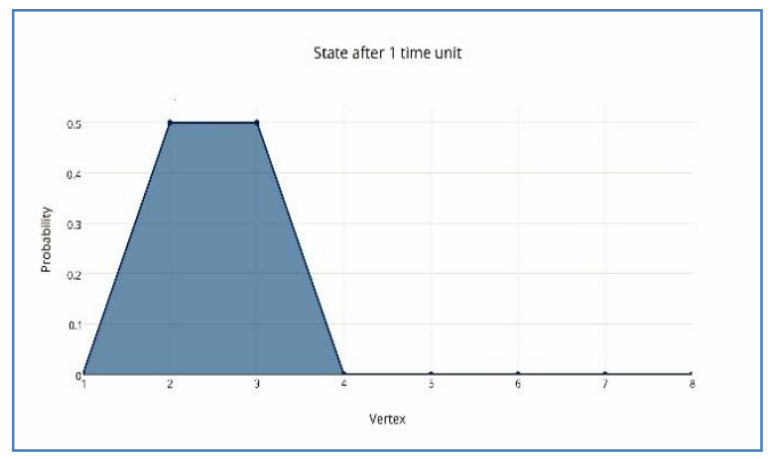

Fig.14. Plot of state vector after 1 time unit.

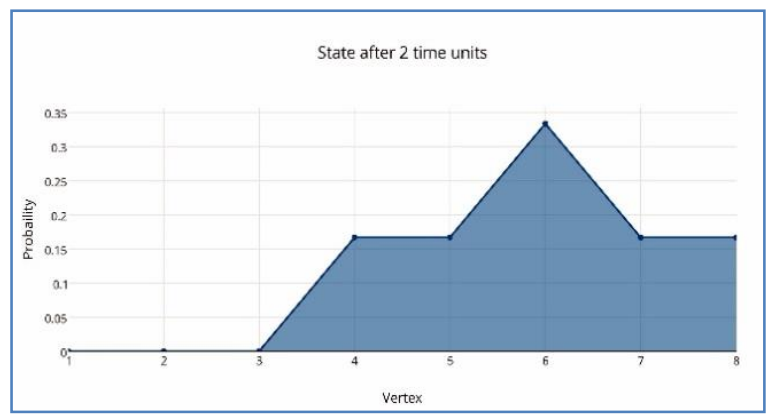

Fig.15. Plot of state vector after 2 time units.

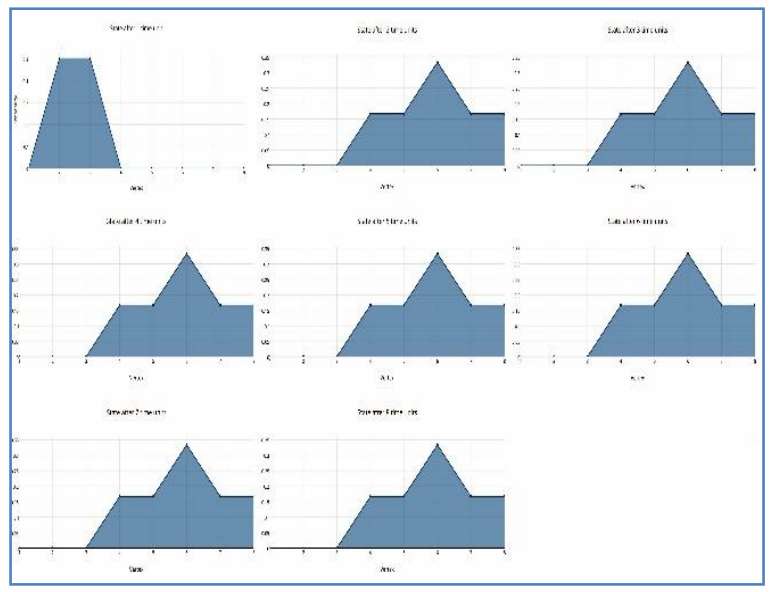

Fig.16. A collage of the plots of state vectors after each time unit

\subsection{Quantum Systems}

By recording the state vector after each time unit (for a total of 8 time units), we plotted the state vector to visualize how the system evolves with time. Figure 17 and 18 shows the state vector after 1 time unit and 2 time units respectively. Figure 19 shows the evolution of the quantum system with time. 


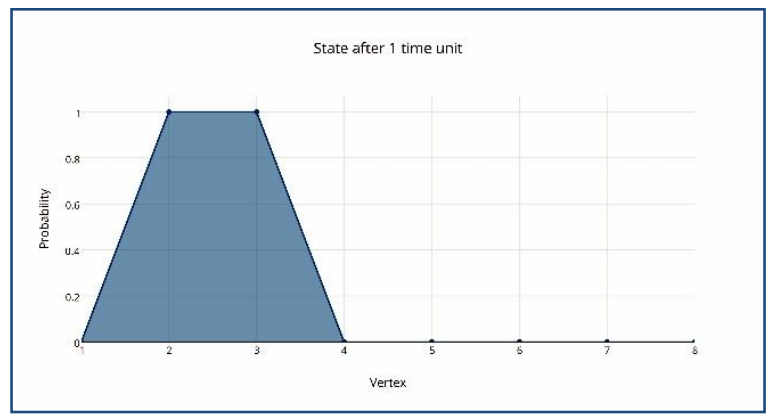

Fig.17. Plot of state vector after 1 time unit.

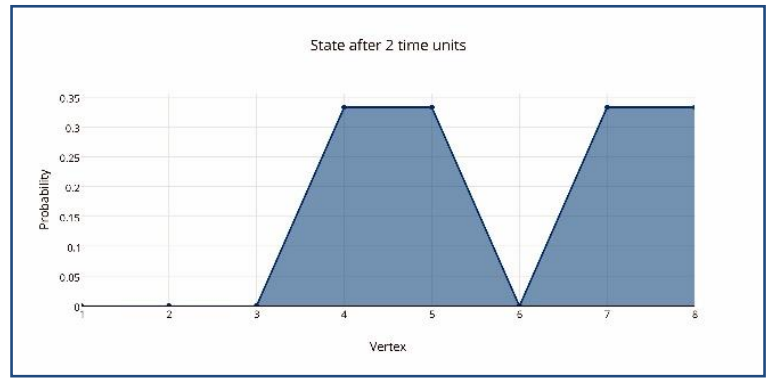

Fig.18. Plot of state vector after 2 time unit.

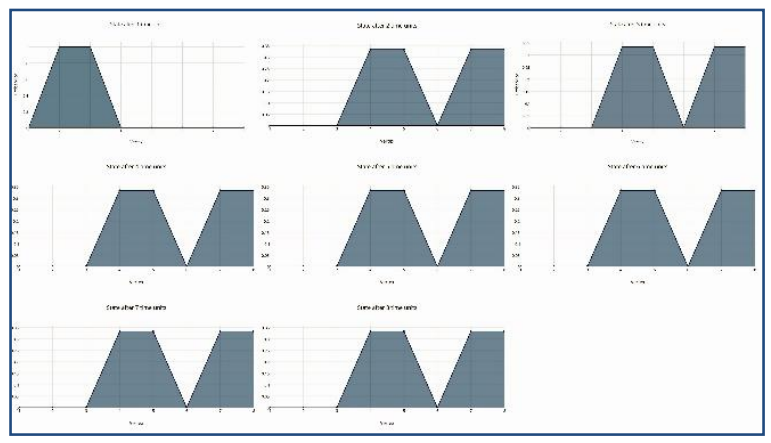

Fig.19. Time evolution of the quantum system after 8 time units.

\section{Results}

The discussion of the results obtained after performing the simulations on of the three systems reveals some interesting facts which are explained in the following sub-sections.

\subsection{Classical Deterministic Systems}

As we already have assumed that for a particle it takes one time unit to travel through a directed edge from one vertex to another changing the overall system state. In our "1-classical-particle, 8-vertex graph" classical deterministic system, vertex 0 has no incoming edge neither has outgoing any edge so the number of particles 
on this vertex will not change or take part in overall system change. This fact can be seen visually in Figure 4.The system evolves with each passing time unit as is evident from Figure 7 through 11 . The particles that leave vertex 1 never reengage themselves at this vertex because no edge is directed towards vertex 1 . We also can see the fact that this system after 3 time units repeats the state because of presence of a loop in the system. This indicates the deterministic nature of the system and we also deduce the fact that the classical systems change in a discrete way that can be understood well.

We understand from system under consideration that the system is deterministic: the system states can be determined easily. We can determine the past system state and can predict the future system state in a deterministic way. The system changes with the time in both in a continuous as well as in stroboscopic manner i.e. the system has discrete and many but comprehendible states [1] (Susskind and Hrabovsky G, 2014).

\subsection{Classical Probabilistic Systems}

As already assumed, the particle takes 1 time unit to go from source to slits and 1 time unit to go from slits to one of the targets. Since there are two paths from the source (vertex 1) to slits (vertex 2 and 3), there is a 50\% probability that the particle will be found at vertex 2 and 50\% probability that the particle will be found at vertex 3, after 1 time unit. This fact is clearly shown in figure 5. In the figure, the "flattened" peak illustrates this fact. Our main focus will be on vertex 6 . At vertex 6 (or target 6), the probability of finding the particle will be maximum. This is because, there are 2 paths to vertex 6 from vertex 1 (via vertex 2 and vertex 3 ). This leads to the fact that the probabilities will get added. Using the principles of the counting [15] (Grimaldi, Ralph P, 2006), we've the probability, $P$ of finding the particle at vertex 6 as:

$$
\begin{gathered}
P(\text { vertex } 6)=P(\text { edge } 1,2) * P(\text { edge } 2,3)+P(\text { edge } 1,3) * P(\text { edge } 3,6) \\
P(\text { vertex } 6)=\frac{1}{2} * \frac{1}{3}+\frac{1}{2} * \frac{1}{3}=\frac{1}{6}+\frac{1}{6}=\frac{1}{3}
\end{gathered}
$$

At other targets, the probability of finding the particle is $1 / 2 * 1 / 3=1 / 6$. This is illustrated in figure 6 . The plot clearly shows that the probability of finding the particle at vertices $(4,5,7$, and 8$)$ is $1 / 6 \approx 0.166$ and at vertex 6 is $1 / 3 \approx 0.333$. This can also be verified by checking the dynamics matrix entry after 2 time units, i.e., $M^{2}$. We find that $M^{2}[1,6]=1 / 3$ which confirms the above facts. The "take-away" lesson is that in classical probabilistic systems, probabilities are always added so as to increase the likelihood of finding the particle at a particular point.

\subsection{Quantum Systems}

In classical probabilistic systems, we use real positive probabilities (between 0 and 1) to calculate the likelihood of finding the particle at some point. However, in Quantum Systems, the probabilities are not given as real numbers but as complex numbers. The modulus square of the complex number or "probability amplitude" gives the probability of finding the quantum mechanical particle, a photon, at a particular point. The reason for introducing complex numbers in Quantum Mechanics is that real probabilities when added increase, while as complex numbers can lower the probabilities when added together [2] (Yanofsky et al, 2008). This is illustrated in figure 10. If we compare figure 7 and 10, the probability of finding the photon at vertex 6 is ZERO (in spite of the fact that there are 2 paths for the photon to reach vertex 6 ) in the latter, while in former the probability is $1 / 3 \approx 0.333$. This can be shown from by applying principle of counting on fig 6 .

$$
P(\text { vertex } 6)=P(\text { edge } 1,2) * P(\text { edge } 2,3)+P(\text { edge } 1,3) * P(\text { edge } 3,6)
$$




$$
P(\text { vertex } 6)=\frac{1+i}{2} * \frac{1-i}{\sqrt{6}}+\frac{1+i}{2} * \frac{-1+i}{\sqrt{6}}=0
$$

This can also be verified by checking the dynamics matrix entry after 2 time units, i.e., $R^{2}$. We find that $R^{2}[1,6]=0$ which confirms the above facts. This can explained only if the probabilities when added together lower the total probabilities. This is possible if we use complex numbers instead of real numbers to represent probabilities. Although, there are 2 paths for the photon to travel to vertex 6 , still no photon reaches there. This is called Quantum Interference. Classical physics fails to explain this because it uses real positive probabilities. The behavior of the Quantum system under consideration is consistent with experiments [10] (Dirac, Paul, 1981), [8] (Feynman Richard P et al, 1993), [16] (Feynman et al, 2011). Quantum theory tells us that single photons enters the 2 slits "simultaneously" and exits the same slits and cancel each other. This is a manifestation of Principle of Superposition. This can be illustrated by a 3-D plot as shown in figure 20. As can be seen in the figure, the photon can be found with probability 1 at vertex 2 and 3 simultaneously at the same time. We can conclude that this experiment (simulation) illustrates the Quantum Interference and Principle of Superposition and also provides an explanation where the Classical physics fails. The ability of a quantum mechanical particle to exist simultaneously in many states at the same time is the real power behind Quantum Computers.

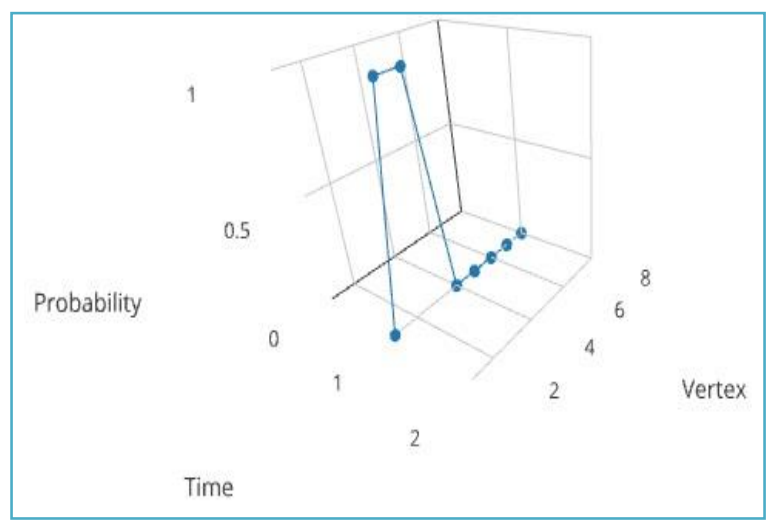

Fig.20. A 3-D plot of the Quantum System after 1 time unit.

\section{Conclusions}

We conclude that classical deterministic systems change with deterministic laws with past and future states calculable given the present state. For classical probabilistic systems, the evolution is governed by the probabilistic laws with in which probabilities when added increase the overall probability. For Quantum system, the probabilities when added, can cancel each other and lower the overall probability. Also, a quantum mechanical particle can exist in multiple states simultaneously at the same time.

\section{References}

[1] Susskind Leonard and Hrabovsky George Classical Mechanics: The Theoretical Minimum [Book]. Clays Ltd, St Ives plc, Great Britain : Penguin, 2014.

[2] Yanofsky, Noson S., Mirco A. Mannucci, and Mirco A. Mannucci. Quantum computing for computer scientists. Vol. 20. Cambridge: Cambridge University Press, 2008. 
[3] Mermin, N. David. "From Cbits to Qbits: Teaching computer scientists quantum mechanics." American Journal of Physics 71.1 (2003): 23-30

[4] Arrighi, Pablo. Quantum computation explained to my mother. No. quant-ph/0305045. 2003.

[5] Bao, Lei, and Edward F. Redish. "Understanding probabilistic interpretations of physical systems: A prerequisite to learning quantum physics." American Journal of Physics 70.3 (2002): 210-217.

[6] Boas, Mary L. Mathematical methods in the physical sciences. Wiley, 2006.

[7] Simulation 18 - Classical probability density [Online]. - QuVis: The University of St Andrews Quantum Mechanics Visualisation project. - Dec 22, 2014. http://www.standrews.ac.uk/physics/quvis/embed_item_3.php?anim_id=18\&file_sys=index_chem.

[8] Feynman Richard P., Leighton Robert B. and Sands Matthew The Feynman Lectures on Physics Vol. 3 [Book]. - [s.l.] : Narosa Publishing House, 1993

[9] Yanofsky, Noson S. "An introduction to quantum computing." Proof, Computation and Agency. Springer Netherlands, 2011. 145-180.

[10] Dirac, Paul Adrien Maurice. The principles of quantum mechanics. No. 27. Oxford university press, 1981.

[11] Robyn The Best Chocolate Cake Recipe [Online] // Add A Pinch. - 2013. - January 5, 2015. http://addapinch.com/cooking/the-best-chocolate-cake-recipe-ever/.

[12] Biamonte Jacob D. Private Communication with Jacob D. Biamonte (Research Group Leader at ISI Foundation, Torino, Italy). - 2014

[13] Earley Clarke $\mathrm{NaCl}$ and the structure of Salts [Online]. - August 19, 2008. - 12 2014, 30. http://www.personal.kent.edu/ cearley/ChemWrld/jmol/crystals/crystals.htm.

[14] Leornard Susskind Classical physical systems [Online] // http://www.lecture-notes.co.uk. - Dec 16, 2010. 12 2014, 30. http://www.lecture-notes.co.uk/susskind/quantum-entanglements/lecture-1/classical-physical-systems/.

[15] Grimaldi, Ralph P. Discrete and Combinatorial Mathematics, 5/e. Pearson Education India, 2006.

[16] Feynman, Richard P., Robert B. Leighton, and Matthew Sands. Six not-so-easy pieces: Einstein's relativity, symmetry, and space-time. Basic Books, 2011.

\section{Authors' Profiles}

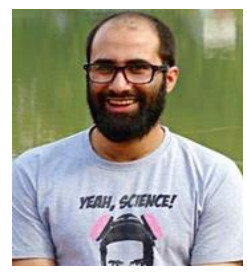

Younis A Shah has completed his B.Tech (CSE) - Islamic University of Science \& Technology - 2011, India and MSc.IT from university of Kashmir India . His Research interests are Quantum computing and Simulation.

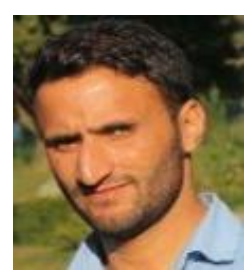

Dr. Irshad A Mir is currently working as Assistant Professor(c) in the Directorate of Information Technology \& Support System for Masters of Sciences in Information Technology (MSc.IT) programme at University of Kashmir, India. He has completed his $\mathrm{PhD}$ in Computer Sciences from the University of Kashmir in 2013 and Masters (MCA) from the same university in 2009. His Area of Interest is Information Security with emphasis on Software Security and evaluation. 


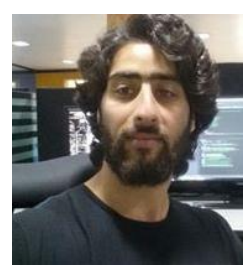

Uzair Mohammad Rather. Bachelors in Computer Science - University of Kashmir - Feb 2010 Masters in Computer Science - University of Kashmir - May 2013.

How to cite this paper: Younis A. Shah, Irshad.A. Mir, Uzair M. Rathea,"Quantum Mechanics Analysis: Modeling and Simulation of some simple systems", International Journal of Mathematical Sciences and Computing(IJMSC), Vol.2, No.1, pp.23-40, 2016.DOI: 10.5815/ijmsc.2016.01.03 